\title{
Radio continuum observations of Comet C/1999 S4 (LINEAR) before, during, and after break-up of its nucleus
}

\author{
W. J. Altenhoff ${ }^{1}$, F. Bertoldi ${ }^{1}$, K. M. Menten ${ }^{1}$, A. Sievers ${ }^{2}$, C. Thum ${ }^{3}$, and E. Kreysa ${ }^{1}$ \\ 1 Max-Planck-Institut für Radioastronomie, Auf dem Hügel 69, 53121 Bonn, Germany \\ e-mail: fbertoldi, ekreysa, kmenten@mpifr-bonn.mpg.de \\ 2 Institute for Radio Astronomy at Millimeter Wavelengths (IRAM) Avenida Divina Pastora 7, 18012 Granada, Spain \\ e-mail: sievers@iram.es \\ 3 Institute for Radio Astronomy at Millimeter Wavelengths (IRAM) Domaine Universitaire de Grenoble, 300 rue de la piscine, \\ 38406 St. Martin d'Hères, France \\ e-mail: thum@iram.fr
}

Received 18 January 2002 / Accepted 21 May 2002

\begin{abstract}
We observed radio continuum emission from Comet C/1999 S4 before, during, and after break-up of its nucleus. The detections before break-up indicate a photometric diameter of $4.7 \mathrm{~km}$, from which we estimate that the nuclear diameter was about $0.9 \mathrm{~km}$. We derive a dust production rate of $\sim 9 \times 10^{4} \mathrm{~g} \mathrm{~s}^{-1}$, corresponding to $0.03 \mathrm{~g} \mathrm{~s}^{-1}$ per square meter of the nuclear surface area, which is comparable to the values found for comets 1P/Halley and C/1995 O1 (Hale-Bopp). The implied gas-to-dust mass production ratio was between 2 and 5. The high daily erosion rate and the continuous fragmentation over months, as derived from water production rates, could not be confirmed. The low radio emission of the fragments directly after the break-up and their low cometary activity may be explained by their low surface temperatures. The published optical and most radio data support that the main nuclear decay started July 23, 2000. Our upper limits on the flux density obtained after the nuclear disintegration put an upper bound on the particulate dust mass released during that event. A scenario in which most of the nucleus disintegrated into many big boulders and part of it into a dust cloud is consistent with our observations.
\end{abstract}

Key words. comets: general - comets: individual: C/1999 S4 - radio continuum: solar system

\section{Introduction}

Radio astronomical research on comets started with the apparition of Comet C/1956 R1 (Arend-Roland), which many of the radio observatories of the time tried to detect - unsuccessfully - at various frequencies (Lovell 1959). Two decades later Hobbs et al. (1975) reported the detection of a strong radio signal from Comet C/1973 E1 (Kohoutek), which was thought to originate from an Icy Grain Halo (IGH; Delsemme 1973; Gibson \& Hobbs 1981, 1983) of cm-sized icy grains. Subsequent attempts to verify this model with observations of other comets failed (e.g., Snyder et al. 1983; Hoban \& Baum 1987). Altenhoff et al. (1983) reported the detection of thermal emission from the nucleus of Comet C/1983 H1 (IRAS-ArakiAlcock). Their results of the nuclear size were consistent, e.g., with the infrared (IR) observations of Hanner et al. (1985) and with radar observations of Harmon et al. (1989), but could not be confirmed by the interferometric radio observations of de Pater et al. (1985). Altenhoff et al. $(1986,1989)$ monitored the

Send offprint requests to: W. J. Altenhoff, e-mail: wja@mpifr-bonn.mpg.de radio emission of Comet $1 \mathrm{P} /$ Halley over several days and measured the extent of the radio halo confirming for the first time the presence of $\mathrm{mm}$-sized ice/dust particles.

More recently the exceptional brightness of Comets C/1996 B2 (Hyakutake) and C/1995 O1 (Hale-Bopp) allowed detailed radio observations. Altenhoff et al. (1999, Paper I), de Pater et al. (1997, 1998), and Jewitt \& Matthews (1997, 1999) demonstrated the potential of radio- and millimeter-wavelength continuum observations for studies of the nuclear diameter and dusty halo. The interpretation of these observations is however complicated by spectral index variation in the halo emission, as seen with the dual frequency interferometer, by uncertainties in the frequency dependence of the absorption coefficient for the differently sized halo dust particles, and by the unexpected dependence on the observation frequency of the halo size (Paper I).

When Comet C/1999 S4 (LINEAR) (short: comet S4) was detected, Marsden 1999 predicted that it would become optically bright. We therefore initiated a series of observations at various radio telescopes, at frequencies ranging from 32 to $860 \mathrm{GHz}$. During these observations the cometary nucleus 
disintegrated, providing an unique opportunity to examine the comet and its composition.

\section{Observations}

First observations of comet S4 were done in March and April 2000 using the $250 \mathrm{GHz}$ Max-Planck Millimeter Bolometer (MAMBO) array at the IRAM $30 \mathrm{~m}$ telescope on Pico Veleta, Spain. MAMBO is a 37-element bolometer array with an effective frequency of $250 \mathrm{GHz}$ and a bandwidth of about $80 \mathrm{GHz}$. Under good weather conditions each bolometer has a sensitivity of about $35 \mathrm{mJy} \mathrm{s}^{-0.5}$ and a $H P B W$ of $10.7^{\prime \prime}$; the pixel separation is about $23^{\prime \prime}$, the instantaneous field of view of the array has a diameter of about $120^{\prime \prime}$.

The observations were done in the standard ON-OFF mode, with individual scans of divided in 12 or 16 subscans of $10 \mathrm{~s}$ on and off source integration, respectively. The secondary mirror was chopped by about 50" in Azimuth at $2 \mathrm{~Hz}$, and the telescope was nodded by the same distance after each subscan. The data were analysed with the MOPSI package, created by Zylka (1998), which allows for efficient sky noise reduction (see Bertoldi et al. 2000). More details on the instrumentation at both telescope sites and the observing and data reduction techniques can be found in Paper I.

The results are compiled in Table 1 . The unusually high non-gravitational forces on comet S4 (if not considered in the ephemerides) can result in position errors up to 36" (Marsden 2000c) and correspondingly to underestimates of the cometary flux density. Therefore the references to the orbital elements used for the observations are included in Table 1.

On March 27, 2000 our trial observations resulted in a marginal detection (Fig. 1 and Table 1), promising a strong signal at $250 \mathrm{GHz}$ near perigee. The attempt to confirm this detection in early April failed probably because of unsufficient sensitivity (Table 1).

Unexpectedly then in July our MAMBO observations failed to detect the comet, either because the signal of comet S4 was dramatically weaker as predicted, or because of problems related to the non-gravitational forces. Our telescope control programs are designed to handle unperturbed orbital elements only for the observing epoch without correction for non-gravitational parameters. Responding to our need Green (2000) provided a set of unperturbed orbital elements, which helped to detect comet S4. Since position errors due to unaccounted non-gravitational parameters vanish at the epoch of the orbital elements, Marsden (2000b) provided orbital elements for 3 epochs near the perigee of comet S4. Using these elements at the nearest epoch (as unperturbed elements) leads to errors smaller 1" (Marsden, private communication). We therefore used these elements. With these elements we could estimate the position error on July 18, it was about $12^{\prime \prime}$; the reason, why we did not find the comet in the instantaneous field of view or in our search map, is the unexpected low signal. Indeed, the observed flux densities were about two orders of magnitude below the predictions made from the trial measurements in March. They were also too weak for halo size and position determinations.
Table 1 summarizes the results of the MAMBO observations. Despite the adverse and variable atmospheric conditions typical for daytime observing in summer, we obtained two fully and one nearly significant detections (Table 1, Fig. 1).

Our measurements at $32 \mathrm{GHz}\left(26^{\prime \prime} F W H M\right.$ beam) using the $100 \mathrm{~m}$ telescope are also included in Table 1 . They were made with the improved new second module of the multifeed system. To reduce confusion, weather, and interference problems, multiple scans were made in the double beam mode (see Paper I).

During the time of our observations, and in fact in the midst of our second $100 \mathrm{~m}$ observation, the cometary nucleus disintegrated (Kidger 2000; Sekanina 2000). For the following observations we used the orbital elements of Marsden (2000b) for the appropriate epoch in the hope that they would describe the fragment's center of gravity.

\section{Discussion}

The initial detection on March 27 is shown in Fig. 1. Assuming a dependence of the $250 \mathrm{GHz}$ flux on heliocentric distance, $r$, and geocentric distance, $\Delta$, following $\propto \Delta^{-2} r^{-0.5}$, we extrapolated the flux measured on March 27 to July 23, to predict a flux density of about $165 \mathrm{mJy}$ near perigee. The signal we actually observed near perigee was much smaller than that (Table 1). On March 27 the proper motion of the comet was low, about 8 " during the integration time of $45 \mathrm{~min}$. A background source accidentally passing the beam could have produced the signal, but the probability for this is very small. Another explanation of the observed signal could be a cometary outburst. Weaver et al. (2001) have observed enhanced, transient optical activity of this comet between July 5 and 7; such optical variability is not unusual for comets. But a corresponding brightening in the radio range has not yet been observed.

The $250 \mathrm{GHz}$ flux obtained on July 20 seems higher than those measured a day earlier or after. These data were taken at a low elevation. The uncertainty of the large gain correction factor of 7.3 may be the cause of the large discrepancy of this measurement. To obtain an average signal we computed the weighted average of the flux densities measured in July before the July 23 nuclear disruption and scaled this flux to the cometary position on July 20, when $\Delta=0.39 \mathrm{AU}$ and $r=0.77 \mathrm{AU}$. For this we again assumed $S_{v} \propto \Delta^{-2} r^{-0.5}$. We then find a weighted average $S_{250 \mathrm{GHz}}=2.94 \pm 0.53 \mathrm{mJy}$, and a $3 \sigma$ upper limit $S_{32 \mathrm{GHz}} \leq 0.5 \mathrm{mJy}$. From these dual frequency measurements we find a limit to the spectral index $\alpha \geq 0.85\left(S_{v} \propto v^{\alpha}\right)$.

\subsection{Nucleus size}

Assuming that the comet halo particles are in thermal equilibrium with insolation we can use the thermal emission to calculate the photometric size of the cometary emission, i.e., the equivalent area of a blackbody at the distance and temperature of the comet emitting the observed flux density. Using our weighted average $S_{250}=2.94 \pm 0.53 \mathrm{mJy}$ for July 20, we derive a photometric diameter, $D_{\mathrm{ph}}=(4.7 \pm 0.4) \mathrm{km}$. In the absence of a particle halo $D_{\text {ph }}$ will be identical with the nuclear diameter, 

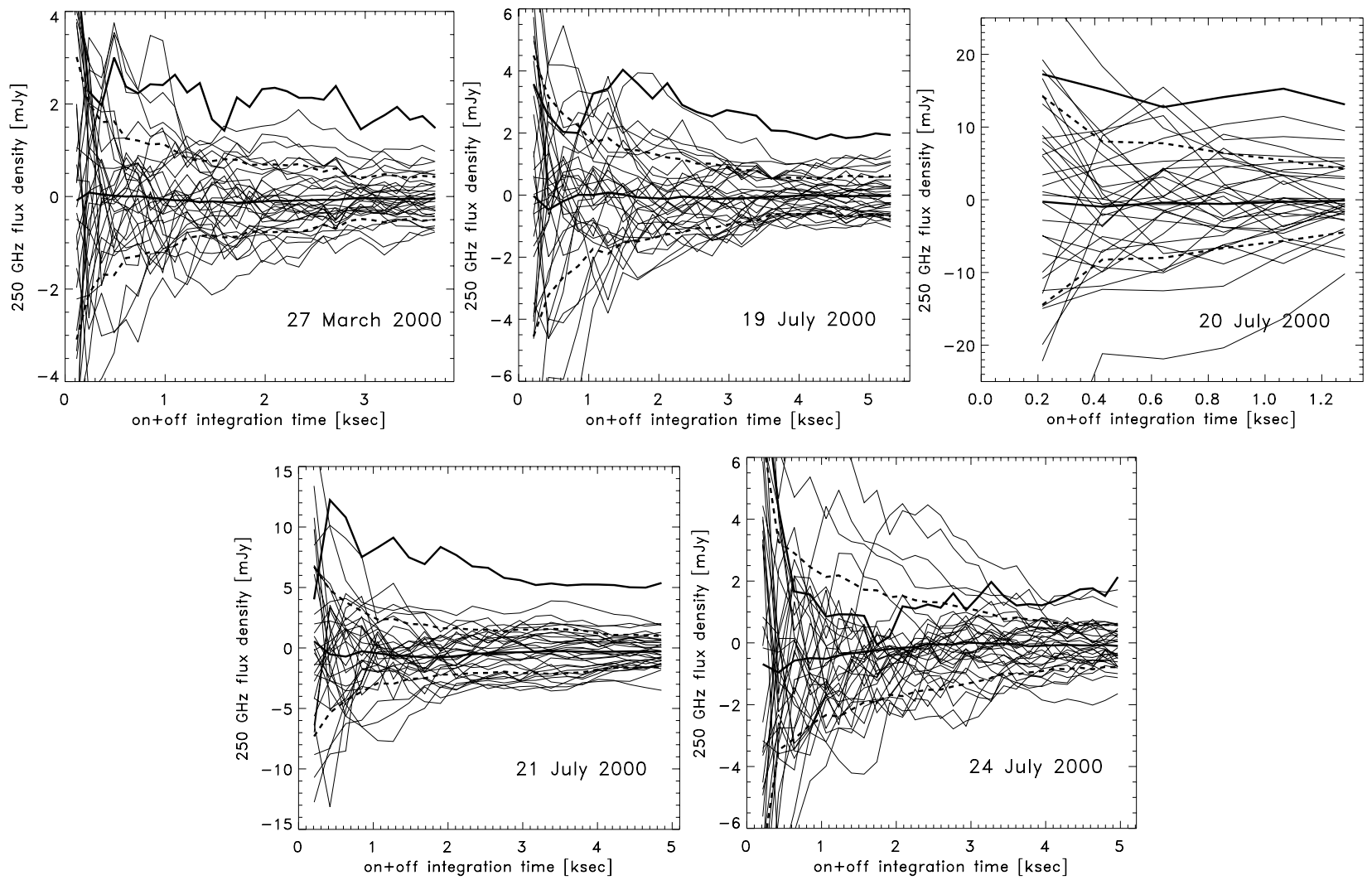

Fig. 1. Observed signals at $250 \mathrm{GHz}$ of Comet C/1999 S4. The time integrated signals are plotted for all bolometer channels as function of integration time. The signal of Channel 1 (on source) is emphasized by the upper thick line. The \pm rms dispersion of the off-source channels is shown as dashed lines. The lower solid line shows the average signal of the off-source channels.

Table 1. Observational results.

\begin{tabular}{|c|c|c|c|c|c|c|c|c|}
\hline $\begin{array}{l}\text { Date } \\
2000\end{array}$ & $\begin{array}{l}\text { UT } \\
{[\mathrm{h}]}\end{array}$ & $\begin{array}{c}r \\
{[\mathrm{AU}]}\end{array}$ & $\begin{array}{c}\Delta \\
{[\mathrm{AU}]}\end{array}$ & $\begin{array}{l}t_{\text {int }} \\
{[\mathrm{s}]}\end{array}$ & $\begin{array}{c}S_{v} \\
{[\mathrm{mJy}]}\end{array}$ & $\begin{array}{l}\text { Epoch } \\
2000\end{array}$ & Orb. elements & Reference \\
\hline \multicolumn{9}{|c|}{$250 \mathrm{GHz}$ MAMBO results from IRAM $30 \mathrm{~m}$ telescope } \\
\hline Mar. 27 & 15.8 & 2.175 & 2.999 & 3711 & $1.56 \pm 0.45$ & Aug. 4.0 & MPEC 37478 & Marsden (2000) \\
\hline Apr. 12 & 12.8 & 1.961 & 2.889 & 1157 & $0.34 \pm 0.77$ & dto. & dto. & dto. \\
\hline Jul. 18 & 6.7 & 0.781 & 0.422 & 3395 & $-1.2 \pm 0.6$ & Aug. 4.0 & MPEC 2000-O02 & Marsden (2000a) \\
\hline Jul. 19 & 6.1 & 0.777 & 0.403 & 5326 & $2.13 \pm 0.55$ & Aug. 4.0 & priv. com. & Green (2000) \\
\hline Jul. 20 & 5.4 & 0.774 & 0.389 & 1281 & $12.90 \pm 4.80$ & Jul. 15.0 & MPEC 2000-O07 & Marsden (2000b) \\
\hline Jul. 21 & 7.5 & 0.771 & 0.378 & 4873 & $5.36 \pm 1.28$ & Jul. 25.0 & dto. & dto. \\
\hline Jul. 24 & 9.9 & 0.766 & 0.379 & 4966 & $1.92 \pm 0.81$ & dto. & dto. & dto. \\
\hline Aug. 4 & 15.5 & 0.787 & 0.670 & 2116 & $-0.69 \pm 1.13$ & Aug. 4.0 & dto. & dto. \\
\hline Aug. 7 & 15.1 & 0.803 & 0.777 & 6140 & $1.64 \pm 0.76$ & dto. & dto. & dto. \\
\hline Aug. 10 & 13.1 & 0.822 & 0.883 & 3818 & $-3.6 \pm 1.96$ & dto. & dto. & dto. \\
\hline \multicolumn{9}{|c|}{$32 \mathrm{GHz}$ data from Effelsberg $100 \mathrm{~m}$ telescope } \\
\hline Jul. 19 & 23.8 & 0.775 & 0.392 & 16000 & $-0.30 \pm 0.22$ & Aug. 4.0 & priv. com. & Green (2000) \\
\hline Jul. 23 & 17.6 & 0.767 & 0.375 & 13500 & $-0.60 \pm 0.27$ & Jul. 25.0 & MPEC 2000-O07 & Marsden (2000b) \\
\hline
\end{tabular}

$D_{\mathrm{n}}$. This is the smallest photometric diameter derived for any active comet detected so far at millimeter wavelengths.

In principle, one can derive the nuclear diameter of a comet at large heliocentric distances from the visual magnitude and the geometric albedo (Jewitt 1991). This method worked well, e.g., for Comet 1P/Halley at a heliocentric distance of 11.04 AU (Jewitt et al. 1982), assuming the standard geometric albedo of $p=0.04$. However, applied to Comet S4 at the time of its detection at $r=4.4 \mathrm{AU}$, the signal of the nucleus was strongly blended by cometary activity, i.e., a contribution by its halo, so that even a rough size determination from optical measurements became impossible.

The unusually large non-gravitational parameters derived by Marsden (2000b) indicate a small nuclear size (Marsden et al. 1973), leading Sekanina (2000) to the suggestion that S4 was a trailing fragment of an earlier comet. 
Marsden et al. (1973) had discussed the relation between nuclear radius, nuclear density, and non-gravitational parameters, but the implied correlations were not yet backed by observations, probably because diameters and densities could not be independently determined with sufficient accuracy. Presently, it is therefore still impossible to obtain a comet size estimate from the effects of non-gravitational forces on its orbit.

In the past, radar observations led to nuclear size determinations for a number of comets (Harmon et al. 1999). Unfortunately, for S4 no such observations were attempted because of the comet's large geocentric distance at perigee.

Since other means to derive a nuclear size are not available, we must rely on the photometric diameter derived from the $250 \mathrm{GHz}$ flux density. In Paper I, photometric and nuclear sizes were determined independently for three comets. On average the nuclear diameter was found to be $20 \%$ of the photometric diameter of these active comets. If we assume that this relation also holds for $\mathrm{S} 4$, we estimate the nuclear diameter, $D_{\mathrm{n}} \approx 0.9 \mathrm{~km}$.

In fact we know that the underlying relation between photometric and nuclear diameter does not hold as a general rule. Radio observation of Comet C/1983 H1 (IRAS-Araki-Alcock) by Altenhoff et al. (1983), combined with radar observations of Harmon et al. (1989) and Goldstein et al. (1984), have shown that its photometric and nuclear size are nearly equal. A similar conclusion was drawn by Jewitt \& Luu (1992) from their photometric observations for Comet C/1989 Q1 (Okazaki-LeviRudenko). Both these comets were "new" objects, but with very little cometary activity.

Another indirect method for estimating the nucleus size was suggested by Keller (1990). He showed that a lower limit to $D_{\mathrm{n}}$ can be derived from the water production rate, $Q_{\mathrm{H}_{2} \mathrm{O}}$, if the nucleus is covered by ice. For Comet Halley he thereby derived a plausible minimum diameter of $6.8 \mathrm{~km}$. For S4, Mäkinen et al. (2001) monitored $Q_{\mathrm{H}_{2} \mathrm{O}}$ with the SWAN camera on the SOHO spacecraft from the end of May through the nuclear break-up to mid August 2000. They derive an average minimum cross section of $\geq 1 \mathrm{~km}^{2}$, corresponding to a minimum diameter of $1.1 \mathrm{~km}$. If a significant part of the $Q_{\mathrm{H}_{2} \mathrm{O}}$ is originating from icy grains in addition to those from the nuclear surface, the minimum diameter may be overestimated.

A substantially smaller diameter may be inferred from observations of the nuclear fragments after breakup. From reassembling the fragments detected in HST and VLT images into a single spherical object Weaver et al. (2001) obtain a nuclear diameter of $\sim 200 \mathrm{~m}$. This number is considered a lower limit since the contribution from fragments smaller than the $\sim 50 \mathrm{~m}$ detection threshold is not known.

It appears that the latter estimates of $D_{\mathrm{n}}$ well bracket the value of $0.9 \mathrm{~km}$ derived from our measurement of the $250 \mathrm{GHz}$ photometric diameter. In what follows, we adopt this value, but emphasize that our estimate is very crude.

\subsection{Disruption of the nucleus}

Between July 23.9 and 24.9 Kidger (2000) observed a highly condensed central optical condensation, whose brightness was decreasing by a factor of about 3 within 24 hours. In the following night the condensation became elongated and the brightness continued to fade. The major axis of this cloud, oriented in tail direction, continued to grow with a velocity of $40 \mathrm{~m} \mathrm{~s}^{-1}$. From the low velocity and from the lack of any subnuclei Kidger concluded that the nucleus had completely disintegrated, forming this particulate cloud. Sekanina (2000) estimated that the event began July 23.6 UT and suggested that the cloud contained submillimeter-sized and larger dust particles sensitive to considerable repulsive acceleration. Mäkinen et al. (2001) and Bockelée-Morvan et al. (2001) argue that the "complete fragmentation" started already with an outburst on July 21 , but we take that the final catastrophic event started with the creation of the highly concentrated and expanding dust cloud (in short, "Kidger cloud").

At this time we were observing the comet at $32 \mathrm{GHz}$ using the $100 \mathrm{~m}$ telescope at Effelsberg (Table 1). The disruption did not leave a trace in our recording, neither a flare-up during the disruption nor any signal afterwards. Our upper limits for the $32 \mathrm{GHz}$ flux densities are derived from night time measurements only.

\subsection{The Kidger-cloud}

In Fig. 2 we sketch the $250 \mathrm{GHz}$ lightcurve of the comet before the disruption and compare the few measurements afterwards with possible models of the disruption. The light curve marked "halo" was calculated as function of $r$ and $\Delta$, see above. The emission of the nucleus is assumed to be $4 \%$ of the total "halo" signal. After the disruption the optical brightness of the halo decreased exponentially (Kidger 2000), due probably to the termination of a steady supply of dust particles to the halo. Our $250 \mathrm{GHz}$ observation on July 24 seems to confirm the decay; after the disruption we did not detect any cometary signal.

A decrease in brightness, similar to that of the Kidger cloud, is seen in the water production observed by Mäkinen et al. (2001), which they relate to a "power law distribution of particles, reflecting the internal structure of the former nucleus".

Kidger (2000) assumed that the nuclear mass was completely dissolved into small particles of unspecified size distribution, which constitute the expanding cloud.

In our model we assume particles of sizes equal to the observing wavelength $(1.2$ and $9 \mathrm{~mm}$ ). If the small particles from inside the nucleus were initially much colder, they would have adjusted to the equilibrium temperature with insolation within minutes. In this paper we assume that the ice/dust particles are at equilibrium temperature, even though recently Peschke et al. (2002) reported on infrared observations of comet S4, which indicate a dust temperature about $20 \%$ below the equilibrium temperature, possibly caused by sublimation of the icy grains. This lower brightness temperature could possibly contribute to our unexpectedly low continuum signal. But for a better comprison with our earlier comet observations we use the equilibrium temperature. Additionally we adopt a mean density of $1 \mathrm{~g} \mathrm{~cm}^{-3}$ for the nucleus and the halo particles, within the 
accepted range of cometary densities between porous ice and refractory (asteroidal type) material.

The velocity reported by Kidger corresponds to an expansion velocity of $20 \mathrm{~m} \mathrm{~s}^{-1}$ in the nucleus-tail direction. The orthogonal expansion velocity is not known. Beginning with the initial flux density of the nucleus at breakup, the expected signal of the opaque cloud will increase proportionally to its apparent solid angle, until the cloud becomes transparent. This happens approximately when it reaches the size of the photometric diameter (a cylinder with this diameter and a height of 2 wavelengths, evenly packed with all the nuclear mass). The photometric diameter, 164 and $450 \mathrm{~km}$, of the expanding cloud is then reached after 1.1 and 3.1 hours, for 32 and $250 \mathrm{GHz}$, respectively. Its signal would then be $60 \mathrm{mJy}$ and $27 \mathrm{Jy}$, respectively. With further increasing size the cloud is transparent, and its total flux density stays constant (ignoring sublimation). When the cloud size exceeds the beam width, the observed flux density per beam decreases. The predicted flux density per beam is shown as a dashed line in Fig. 2. From the observed upper limit of the flux density at $250 \mathrm{GHz}$ after the nuclear break up we derive that the mass contained in mm-sized dust grains in the Kidger cloud is $\leq 4 \times 10^{10} \mathrm{~g}$. Our observations are insensitive to submillimeter sized dust particles; therefore their contribution to the mass of the Kidger cloud cannot be estimated.

Weaver et al. (2001) show the "cloud" of the re-appearing fragments for August 5; this can be compared to the size of the Kidger cloud, extrapolated to this epoch. Ignoring differential non-gravitational forces on the fragments, which all show very little cometary activity, their expansion velocity is about $30 \%$ of the expansion of the Kidger cloud! These high expansion velocities, possibly indicative of high mechanical stresses inside the former nucleus, and the little mass of particulate dust/ice in the Kidger cloud seem to be arguments against a low density in comet S4.

\subsection{Ice particle halo inferred from molecular spectroscopy}

Based on Hubble Space Telescope observations (Weaver et al. 2001) and on observations with the SWAN instrument on board of the SOHO spacecraft Mäkinen et al. (2001) modelled the water production rate of Comet S4 over the period from May to August 2000. They obtain an initial (May 25) mean nuclear diameter of $0.75 \mathrm{~km}$ and a daily nuclear erosion rate of $3.3 \mathrm{~m}$. From this high erosion rate, which is nearly two orders of magnitude higher than what is known for other comets, the authors concluded that "material was leaving the surface by fragmentation rather than by sublimation".

To test this suggestion we used their observed mean daily water mass loss of $4.8 \times 10^{10} \mathrm{~g}$, and the nuclear diameter of $0.75 \mathrm{~km}$ to derive a daily surface erosion of $2.5 \mathrm{~cm}$. This value is within a factor 3 of our measurement (see Sect. 3.6 and Table 2). It is also much closer to the erosion rates of previous comets, and in particular, it does not require continuous fragmentations over months to yield the observed water production rate. We therefore do not see a need to invoke a selective "fragmentation" by mm-size particles of the nucleus.

Near the comet's perigee Bockelée-Morvan et al. (2001) observed a number of molecular lines from which they deduced equivalent water production rates. Comparing these production rates with the corresponding values given in Table 1 by Mäkinen et al. (2001), we find systematic differences. The production rates observed with the SWAN instrument (spatial resolution $1 \mathrm{deg}$ ) showed a constant value within a factor of 2 in the weeks before the disruption on July 23. This is hardly an indication of a fatal decay. But the $Q_{\mathrm{H}_{2} \mathrm{O}}$ determinations of Bockelée-Morvan et al. (2001) with the Pico Veleta telescope (spatial resolution for HCN 9") in the same period showed bigger deviations from the mean value; this could possibly be explained by the different spatial and time resolutions (production rates, each integrated over the beam). But after the disruption both sets of $Q_{\mathrm{H}_{2} \mathrm{O}}$ diverge more strongly. The simplest explanation would be, that the $\mathrm{HCN}$ observations are integrations on the cometary position only and the HCN cloud is expanding and the signal is reduced by dilution. We have considered two possibilities: an expansion velocity of $20 \mathrm{~m} \mathrm{~s}^{-1}$ (Kidger cloud) and $7 \mathrm{~m} \mathrm{~s}^{-1}$ (fragment cloud). In the first case we obtain a good reconciliation of both sets of production rates.

\subsection{Expected continuum emission of the fragments}

Ten days after the (major) break-up Weaver et al. (2000a, 2001) observed that the comet had split into at least 17 massive fragments. At this time the gas and dust production had also partially resumed. For our schematic Fig. 2, showing the expected $250 \mathrm{GHz}$ flux density evolution, we assumed 20 fragments at equilibrium temperature. Geometrically these fragments have a total cross section higher by a factor 2.7 compared with the original nucleus, leading to a corresponding increase of the flux density. If our assumption of a fixed ratio of nuclear and total emission is correct, the total (halo) signal should increase accordingly (allowing for some time delay for generation of the halos). But instead of an increase we see a decrease of the $250 \mathrm{GHz}$ signal on July 24 (and the following observations).

If the fragments contain most of the nuclear mass, the decrease of the $250 \mathrm{GHz}$ emission implies that the brightness temperature of the fragments was much lower than the equilibrium temperature. Indeed, we might expect the inner cometary material, and thereby the initial fragments, to have a temperature comparable to that of outer solar system objects, $\sim 30 \mathrm{~K}$. Such a heating has not been observed at radio wavelengths. But one can get an idea about the time scale from the observed radio light curves of the Moon (see Kuzmin \& Salomonovich 1966) and Mercury (see Morrison 1970) between 8 and $100 \mathrm{GHz}$; Near $100 \mathrm{GHz}$ both objects show a radio phase delay of about 20 degrees, corresponding to a time delay of 2.4 days. A rough extrapolation to $250 \mathrm{GHz}$ would yield a delay between 1.0 to 1.5 days. The heating up of the nucleus in Fig. 2 is a schematic plot of this time scale.

This scenario seems to agree with observations. At the initial low temperature the fragments can notform halos, so 


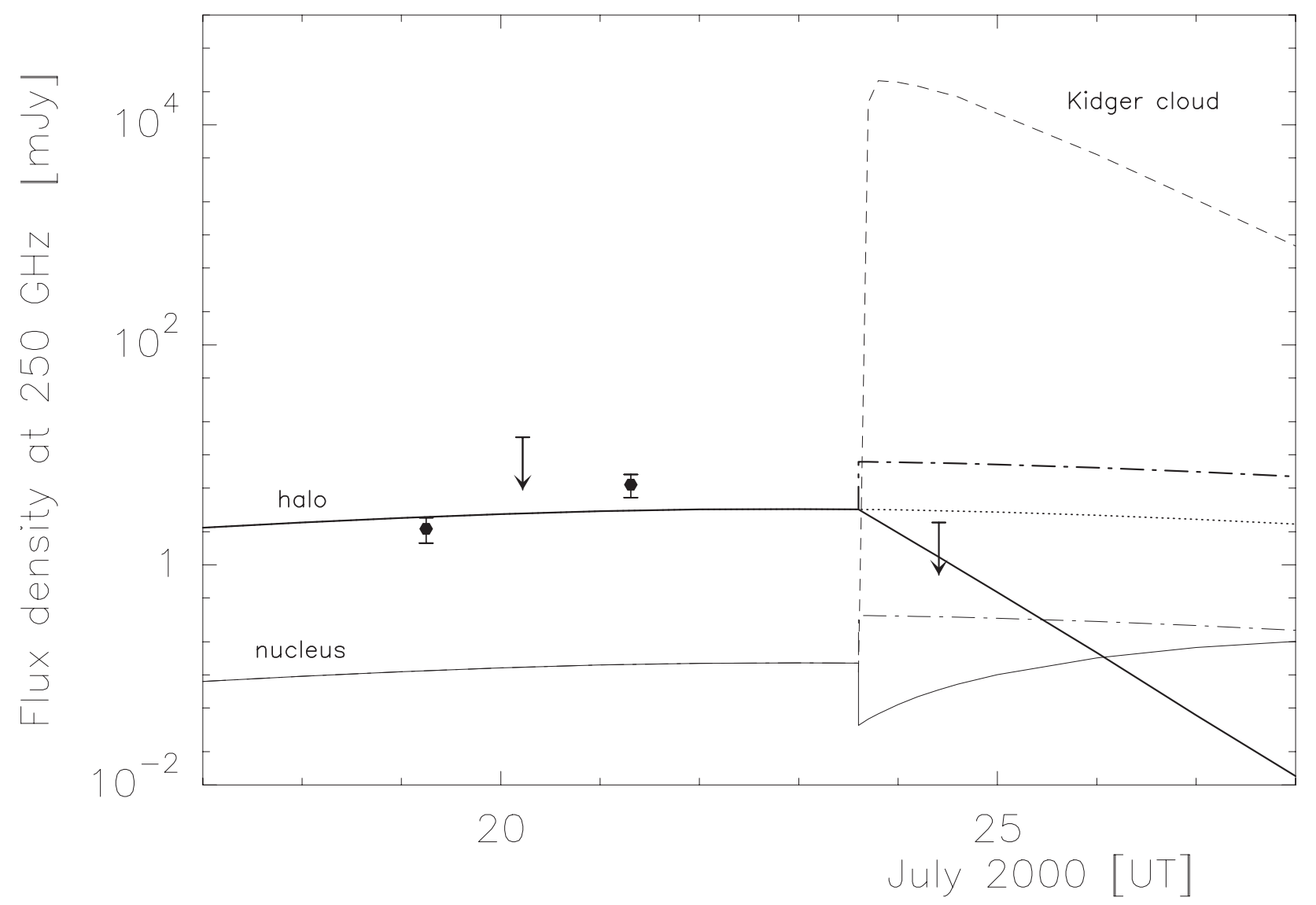

Fig. 2. The observed $250 \mathrm{GHz}$ emission of comet C/1999 S4 through nuclear disintegration on July 23.6 compared with schematic model assumptions. A lightcurve (heavy solid line) for the comet emission before break-up is fitted to the MAMBO observations, the significant detections are shown with their $1 \sigma$ error bars, the insignificant "detections" as upper limits. The extrapolated lightcurve is shown by a dotted line. After break-up (assumed: 20 equal fragments in thermal equilibrium with insolation) the signals of the sub-nuclei and their halos should increase by a factor 2.7 (thin and heavy dash-dot lines, respectively) according to the postulated emission ratio of halo and nucleus; in reality the creation of the halos will take some time, the low emission is partially compensated by that of the halo remnant. The calculated signal of the expanding particulate Kidger cloud containing all the nuclear mass is labeled "Kidger cloud". Probably the radio emission per beam decayed exponentially after the break-up (sloping bold line) like the optical signal of the Kidger cloud (Kidger 2000). The non-detection of any radio emission after the beak-up could possibly be explained by (1) a low surface temperature of the fragments (about $30 \mathrm{~K}$ inside the former nucleus), rising to equilibrium temperature several days later (schematically shown by a thin solid line) and (2) a small mass fraction of mm sized particles in the Kidger cloud.

that the fragments will be invisible optically and at radiowavelengths. After several days some fragments became optically visible (Weaver et al. 2000a) again due to their low level cometary activity. These fragments were at least partially covered with ice (Weaver et al. 2001), others may consist totally out of refractory material (Mäkinen et al. 2001). The low temperature of the fragments and the slow heating may explain also that the water production did not increase after the breakup, but dropped instead, as seen in the works of Mäkinen et al. (2001) and Bockelée-Morvan et al. (2001). The source of the exponentially decreasing water production after July 23 may be the former active dust cover of the nucleus, which may have made up most of the Kidger cloud. This could explain why the Kidger cloud can be used to reconcile the difference between the low and high resolution data of the water production rate (see above).

\subsection{Mass of the nucleus and halo}

Our estimate for the nuclear diameter, $0.9 \mathrm{~km}$, implies a nuclear mass $M_{n}=4 \times 10^{14} \mathrm{~g}$. All information pertaining to the mass of comet $\mathrm{S} 4$ are collected in Table 2.

Our estimate of the mass of the dust particle halo is based on a poorly constrained spectral energy distribution at long ( $\lambda \geq 300 \mu \mathrm{m})$ wavelengths. A long wavelength spectral index, $\alpha=2.8$, measured for comet Hale-Bopp (Paper I; Jewitt et al. 1999) would be compatible with a lower limit of 0.8 we find between $32 \mathrm{GHz}$ and $250 \mathrm{GHz}$ (Sect. 2), and also with the few measurements (Paper I) made for comet Hyakutake.

Following the procedure of Paper I and using the same dust mass absorption coefficient, $\kappa(1.3 \mathrm{~mm})=75 \mathrm{~cm}^{2} \mathrm{~g}^{-1}$, and grain size distribution, $\frac{\mathrm{d} n(a)}{\mathrm{d} a} \propto a^{-3.9}$, and assuming the particles to be at the $r=0.77$ AU equilibrium temperature of $320 \mathrm{~K}$, we obtain a dust mass of $M_{\mathrm{h}}=2.3 \times 10^{9} \mathrm{~g}$ for the $\mathrm{S} 4$ inner $11^{\prime \prime}$ diameter 
Table 2. Derived characteristics of comet C/1999 S4.

\begin{tabular}{ll}
\hline \hline photometric diameter at $250 \mathrm{GHz}$ & $4.7 \pm 0.4 \mathrm{~km}$ \\
Nucleus & \\
$\quad$ diameter & $\sim 0.9 \mathrm{~km}$ \\
$\quad$ mass, $M_{\mathrm{n}}$ & $4 \times 10^{14} \mathrm{~g}$ \\
$\quad$ erosion rate & $1.0 \mathrm{~cm} / \mathrm{day}$ \\
Halo & \\
$\quad$ mass, $M_{\mathrm{h}}$ & $2.3 \times 10^{9} \mathrm{~g}$ \\
$\quad$ dust production rate, $Q$ & $9 \times 10^{4} \mathrm{~g} \mathrm{~s}^{-1}$ \\
$\quad$ water to dust mass ratio & 4 \\
\hline
\end{tabular}

particle halo. The halo thus has a mass which is small, $6 \times 10^{-6}$, compared with the mass of the nucleus. It corresponds to a nucleus surface layer that is only $0.5 \mathrm{~mm}$ thick. The nucleus continuously replenishes the halo, which expands into interplanetary space. With the adopted particle size spectrum, a large fraction of the halo mass is in large grains which are expected to expand at a constant velocity $v \approx 60 \mathrm{~m} \mathrm{~s}^{-1}$ (Crifo 1987). A large particle then resides 7.4 hours in our $11^{\prime \prime}$ beam. On July 20 the comet's dust production (i.e. ejection) rate, $Q$, was therefore $\sim 9 \times 10^{4} \mathrm{~g} \mathrm{~s}^{-1}$, and the rate by which dust particles were lost from each square meter of nuclear surface, $Z \sim 0.03 \mathrm{~g} \mathrm{~m}^{-2} \mathrm{~s}^{-1}$.

The specific dust production rate, $Z$, is a useful quantity for comparing the activity levels of comets. From the dust production rates and the nuclear diameters given in Paper I, we derive $Z=0.01$ for comets Halley $(r=1.12)$ and Hale-Bopp $(r=0.95)$, and $Z=0.3$ for comet Hyakutake $(r=1.02)$. Normalizing these rates to $r=1 \mathrm{AU}$ assuming $Z \propto r^{-1.1}$ (Paper I), comet S4, for which we derived $Z \approx 0.03$, shows similar activity as Hale-Bopp and Halley, while Hyakutake is found to be much more active.

The gas production of S4 was monitored during most of July with several telescopes (Mäkinen et al. 2001; BockeléeMorvan et al. 2001). Biver et al. (2000) report that the derived water production rate, $Q_{\mathrm{H}_{2} \mathrm{O}}$, was strongly variable before the break-up of the nucleus, with values of $3 \times 10^{28} \mathrm{~mol} \mathrm{~s}^{-1}$ during July 6-9 and much lower values during July 12-20, when our observations were made. Shortly after break-up, Schleicher \& Woodney (2000) reported $Q_{\mathrm{H}_{2} \mathrm{O}}=4 \times 10^{27} \mathrm{~mol} \mathrm{~s}^{-1}$. Adopting a mean $Q_{\mathrm{H}_{2} \mathrm{O}}$ of $1 \times 10^{28} \mathrm{~mol} \mathrm{~s}^{-1}$, i.e. $3 \times 10^{5} \mathrm{~g} \mathrm{~s}^{-1}$ of water, for the time of our observations, we obtain a pre-breakup waterto-dust mass production ratio of 4 , and total (dust and water) nucleus erosion rate of about $1 \mathrm{~cm}$ per day.

\section{Summary}

During several observations with the MAMBO bolometer array at the IRAM 30m telescope we detected thermal emission from comet C/1999 S4. Our data imply that before the comet's breakup on 23 July 2000, its radio photometric diameter was $4.7 \mathrm{~km}$ and its nucleus had a diameter of about $0.9 \mathrm{~km}$. Comet $\mathrm{S} 4$ is the smallest comet ever detected at radio wavelengths.

Optical observations had shown that after the comet's break-up a bright, compact dust cloud appeared which expanded and faded with time. After the breakup we were unable to detect the comet's radio emission, supporting the implication of the optical observations that the dust production was reduced.

Our radio observations allow to estimate upper limits of the mass of mm-sized particles in the (Kidger) dust cloud and to consider the changing brightness temperatures of the new fragments.

Acknowledgements. We would like to thank Dr. H. Ungerechts of IRAM for help with early trial measurements, Dr. D. W. E. Green at the CfA for providing undisturbed orbital elements, Dr. B. G. Marsden at the CfA for advice on the usage of orbital elements combined with non-gravitational parameters, Dr. J. Crovisier of Observatoire de Paris-Meudon for new informations and helpful advice, Dr. J. Schmidt at MPIfR for help with Dr. Stumpff's n-body-programme, and Drs. J. Neidhoefer and W. Reich at MPIfR for introduction to the not yet commissioned new module of the $32 \mathrm{GHz}$ system.

\section{References}

Altenhoff, W. J., Batrla, W., Huchtmeier, W. K., et al. 1983, A\&A, $125, \mathrm{~L} 19$

Altenhoff, W. J., Huchtmeier, W. K., Schmidt, J., et al. 1986, A\&A, 164,227

Altenhoff, W. J., Huchtmeier, W. K., Kreysa, E., et al. 1989, A\&A, 222,323

Altenhoff, W. J., Bieging, J. H., Butler, H. M., et al. 1999, A\&A, 348, 1020 (Paper I)

Bertoldi, F., Carilli, C. L., Menten, K. M., et al. 2000, A\&A, 360, 92

Biver, N., Bockelée-Morvan, D., Colom, P., et al. 2000, AAS/Division of Planetary Sci. Meet., vol. 32, 4115

Bockelée-Morvan, D., Biver, N., Moreno, R., et al. 2001, Science, 292, 1339

Crifo, J. F. 1987, in Symp. on the diversity and similarity of comets, Proc. of an Int. Symp., Brussels, ed. E. J. Rolfe, \& B. Battrick, ESA SP, 278, 399

Delsemme, A. H. 1973, Space Sci. Rev., 15, 89

de Pater, I., Wade, C. M., Houpis, L. L. F., \& Palmer, P. 1985, Icarus, 62,349

de Pater, I., Snyder, L. E., Mehringer, D. M., et al. 1997, Planet. Space Sci., 45, 731

de Pater, I., Forster, J. R., Wright, M., et al. 1998, AJ, 116, 987

Gibson, D. M., \& Hobbs, R. W. 1981, ApJ, 248, 863

Gibson, D. M., \& Hobbs, R. W. 1983, ApJ, 269, 805

Goldstein, R. M., Jurgens, R. F., \& Sekanina, Z. 1984, AJ, 89, 1745

Green, D. W. E. 2000, private communication

Hanner, M. S., Aitken, D. K., Knacke, R., et al. 1985, Icarus, 62, 97

Harmon, J. K., Campbell, D. B., Hine, A. A., et al. 1989, ApJ, 338, 1071

Harmon, J. K., Campbell, D. B., Ostro, S. J., \& Nolan, M. C. 1999, Planet. Space Sci., 47, 1409

Hoban, S., \& Baum, S. 1987, Icarus, 70, 264

Hobbs, R. W., Maran, S. T., Brandt, J. C., et al. 1975, ApJ, 201, 749

Jewitt, D. C., Danielson, J. E., Gunn, J. A., et al. 1982, IAU Circ., 3737

Jewitt, D. C. 1991, in Comets in the Post-Halley Era, vol. I, ed. Newburn, R. L. Jr. et al. (Kluwer Acad. Publ.), 19

Jewitt, D. C., \& Luu, J. 1992 Icarus, 100, 187

Jewitt, D. C., \& Matthews, H. E. 1997, AJ, 113, 1145 
Jewitt, D. C., \& Matthews, H. E. 1999, AJ, 117, 1056

Keller, H. U. 1990, in Physics and Chemistry of Comets, ed. W. F. Huebner (Springer, Berlin), 18

Kidger, M. 2000, IAU Cir., 7467, 7472, 7474

Kuzmin, A. D., \& Salomonovich, A. E. 1966, Radioastronomical Methods of Antenna Measurements (Academic Press, NY)

Lovell, A. C. B. 1959, in Paris Symp. on radio astronomy, ed. R. N. Bracewell (Stanford University Press, USA), IAU Symp., 9, 75

Mäkinen, J. T. T., Bertaux, J.-L., Combi, M. R., \& Quemérais, E., 2001, Science, 292, 1326

Marsden, B. G., Sekanina, Z., \& Yeomans, D. K. 1973, AJ, 78, 211

Marsden, B. G. 1999, M. P. E. C., 1999-U14

Marsden, B. G. 2000, M. P. C., 37478

Marsden, B. G. 2000a, M. P. E. C., 2000-O02
Marsden, B. G. 2000b, M. P. E. C., 2000-O07

Marsden, B. G. 2000c, IAU Circ., 7449

Morrison, D. 1970, Space Sci. Rev., 11, 271

Peschke, S. B., Lisse, C. M., et al. 2002, in Cometary Science after Hale-Bopp, IAU Coll., 186, Tenerife January 2002, in preparation Schleicher, D., \& Woodney, L. 2000, IAU Circ., 7475, 7477

Sekanina, Z. 1997, A\&A, 318, L5

Sekanina, Z. 2000, IAU Circ., 7471

Snyder, L. E., Palmer, P., \& Wade, C. M. 1983, ApJ, 269, L21

Weaver, H., \& West, R. 2000, IAU Circ., 7476

Weaver, H. 2000, IAU Circ., 7461

Weaver, H., Sekanina, Z., \& Toth, I., et al. 2001, Science, 292, 1329

Zylka, R. 1998, MOPSI, mapping software, ITA, Heidelberg 